\title{
AN INTERSECTION PROPERTY FOR CONES IN A LINEAR SPACE
}

\author{
R. E. FULLERTON ${ }^{1}$
}

1. Introduction. The author proved in a previous paper [2] that if $X$ is a real linear space with a very rudimentary topology and if $\mathcal{F}$ is the family of all translates of a set $C$ with an extreme point such that the intersection of any two sets in $\mathcal{F}$ is another set in $\mathcal{F}$ then $C$ is a convex cone. A partial converse of that theorem is given in this note. It is shown that if $C$ is a cone and if $x, y \in X$ exist such that $(x+C) \cap(y+C)$ is a cone $K$, then there exists a $z \in X$ such that $K=z+C$. In $\$ 3$ examples of cones $C$ having this property are discussed.

Let $X$ be a linear space with real scalars. A straight line through the origin $\theta$ is defined as the set of all elements $\{\gamma x\},-\infty<\gamma<\infty$ where $x$ is any point of the set $x \neq \theta$. Straight lines not through the origin will be defined as translates of lines through $\theta$. Alternatively, a line joining $x$ and $y$ in $X, x \neq y$ can be defined as the set $\{\alpha x+(1-\alpha) y \mid-\infty<\alpha<\infty\}$. The ray from $x$ through $y$ is the set $\{\alpha y+(1-\alpha) x \mid \alpha \geqq 0\}$. The segment joining $x$ and $y$ is the set $\{\alpha y+(1-\alpha) x \mid 0 \leqq \alpha \leqq 1\}$. A subset $A \subset X$ is linearly closed in $X$ [2] if every line through a point of $A$ intersects $A$ in a line, ray, segment or a single point. Complements of such sets are linearly open. A hyperplane in $X$ is a maximal proper linearly closed subspace of $X$. A cone $C$ in $X$ with vertex $\theta$ is a set such that if $x \in C, \lambda x \in C$ for all $\lambda \geqq 0$. We shall make extensive use of this geometric terminology and open and closed sets in $X$ refer to the quasi topology which has been defined above.

\section{The principal theorem.}

Theorem 1. Let $C$ be a closed convex cone with vertex $\theta$ in a linear space $X$. Let $x, y \in X, x \neq y$ have the property that there exists a $z \in X$ and a cone $K$ with vertex at the origin such that $(x+C) \cap(y+C)$ $=z+K$. Then $K=C$.

Proof. By the homogeneous structure of $X$ it may be assumed that $x=\theta$ and that $C \cap(y+C)=z+K$. Evidently $C \cap(y+C)$ is closed

Presented to the Society August 29, 1957; received by the editors December 18, 1957.

1 This research was supported by the United States Air Force through the Air Force Office of Scientific Research of the Air Research and Development Command, under contract No. AF 18(603)-78. Reproduction in whole or in part is permitted for any purpose of the United States Government. 
and convex. Also by the convexity of $C$, since $z \in C$ and $z \in y+C$, $z+C \subset C \cap(y+C)=z+K$. Thus it must be shown that $z+C \supset C \cap(y+C)=z+K$. Assume that $x^{\prime} \in X$ exists with $x^{\prime} \in z+K$, $x^{\prime} \notin z+C$. Then there exists an open set $N$ with $x^{\prime} \in N, N \cap(z+C)$ void since $z+C$ is closed and $x^{\prime} \notin z+C$. Since $C$ is a cone, for any $\alpha>0, \alpha C=C$ and $\alpha y+C=\alpha y+\alpha C=\alpha(y+C)$. Thus $C \cap(\alpha y+C)$ $=(\alpha C) \cap \alpha(y+C)$. However, by an elementary argument, $(\alpha C) \cap \alpha(y+C)=\alpha[C \cap(y+C)]=\alpha(z+K)$ and $\alpha(z+K)$ $=\alpha z+\alpha K=\alpha z+K$ since $K$ is a cone and $K=\alpha K$ for $\alpha>0$. Thus $C \cap(\alpha y+C)=\alpha z+K$ and $C \cap(\alpha y+C)$ is a cone for any $\alpha>0$. Let $N_{\theta}=N-x^{\prime}$ and let $\alpha>0$ be chosen sufficiently small to insure that $\alpha z \in N_{\theta}, \quad \alpha(y-z) \in N_{\theta}$. Since $x^{\prime} \in z+K, \quad x^{\prime \prime}=x^{\prime}+(\alpha-1) z \in(\alpha-1) z$ $+(z+K)=\alpha z+K$. Also

$\left(x^{\prime \prime}+N_{\theta}\right) \cap(\alpha z+C)=\left(x^{\prime}+(\alpha-1) z+N_{\theta}\right) \cap(z+(\alpha-1) z+C)$

is void since the sets $x^{\prime}+N_{\theta}$ and $z+C$ have a void intersection by the choice of $N=x^{\prime}+N_{\theta}$. Since $C \cap(\alpha y+C)=\alpha z+K, x^{\prime \prime} \in C \cap(\alpha y+C)$ and $x^{\prime \prime} \in C, x^{\prime \prime} \in \alpha y+C$. However, for any point $u \in C$, $\left(u+N_{\theta}\right) \cap(\alpha z+C)$ is nonvoid since $\alpha z+u \in \alpha z+C$ and $(\alpha z+u)-u$ $=\alpha z \in N_{\theta}$. Thus $(\alpha z+u) \in u+N_{\theta}$ and $\alpha z+u \in\left(u+N_{\theta}\right) \cap(\alpha z+C)$. Since $x^{\prime \prime} \in \alpha z+K C C, \quad\left(x^{\prime \prime}+N_{\theta}\right) \cap(\alpha z+C)$ is nonvoid and since $\left(x^{\prime \prime}+N_{\theta}\right) \cap(\alpha z+C)=\left[x^{\prime}+(\alpha-1) z+N_{\theta}\right] \cap[z+(\alpha-1) z+C],\left(x^{\prime}+N_{\theta}\right)$ $\cap(z+C)=N \cap(z+C)$ is nonvoid contrary to assumption. Thus $x^{\prime} \in z+C$ and $z+K C z+C$. Hence $K=C$.

A $C$-cone [1] is a cone having the property that for any $x, y \in X$, there exists $z \in X$ with $(x+C) \cap(y+C)=z+C$. Thus the previous theorem gives rise to the following corollary.

Corollary. If $C$ is a closed cone such that for any two elements $x, y \in X(x+C) \cap(y+C)$ is a cone, then $C$ is a C-cone.

Theorem 1 also yields slight generalizations of the characterization theorems for $L$ and $C$ spaces given in [3] and [1]. In these theorems the hypotheses that the cones which characterize the spaces be $C$ cones can be replaced by the slightly less restrictive hypotheses of the preceding corollary.

3. Examples of cones with property $T$.

Definition. A cone $C$ with vertex $\theta$ has property $T$ if there exists $x \notin C \cup(-C)$ and $y \in X$ such that $C \cap(x+C)=y+C$.

Certain cones having property $T$ are investigated in this section and an example of the use of such a cone in the structure of function spaces is given. A more complete investigation of the structure of such cones and their applications is planned in a subsequent paper. 
Theorem 2. Let $H$ be a hyperplane in $X$ and let $C_{H}$ be any closed convex cone in $H$ with vertex $\theta$. Let $x \in X \backslash H$ and let $r$ be the ray from $\theta$ through $x$. Let $C$ be the convex set determined by $r$ and $C_{H}$. Then $C$ is a closed convex cone such that if $y \in C_{H}, y \neq \theta$, and $L$ is the two space determined by $\theta, x, y$, then for any $u \in L$ there exists $z \in L$ with $C \cap(u+C)$ $=z+C$.

Proof. If $u \in C \cup(-C)$ the conclusion is obvious and $z=u$ or $z=\theta$. Assume $u \notin C \cup(-C)$ and let $r^{\prime}$ be the ray from $\theta$ through $y$. By elementary arguments it can be seen that either $(u+r) \cap r^{\prime}$ or $\left(u+r^{\prime}\right) \cap r$ is nonvoid. By making a translation of the vertex and an interchange of $C$ and $u+C$ if necessary, it can always be assumed that $\left(u+r^{\prime}\right) \cap r$ is nonvoid. Let $z=\left(u+r^{\prime}\right) \cap r$. Then $z \in C \cap(u+C)$. It can easily be established by elementary arguments that if $r^{\prime \prime}$ is a ray lying entirely in $C$ then any translate of $r^{\prime \prime}$ by a point of $C$ also lies in $C$. Making use of this fact, we show that $C \cap(u+C)$ is a cone and hence by Theorem 1, a translate of $C$. Let $w \in C \cap(u+C)$. By construction of $C$ this implies the existence of real numbers $\alpha$, $0 \leqq \alpha \leqq 1$ and $\gamma>1$ such that $w=\alpha v+(1-\alpha) v^{\prime}$ where $v=\gamma z$ and $v^{\prime} \in C_{H}$. If it can be shown that $w-z \in C$ then the ray from $\theta$ through $w-z \in C$ and hence the ray from $z$ through $w$ is in $C$. However, $w=\alpha \gamma z+(1-\alpha) v^{\prime}, w-z=\alpha \gamma z+(1-\alpha) v^{\prime}-z=(\alpha \gamma-1) z+(1-\alpha) v^{\prime}$. Let $v^{\prime \prime}=(1 / \gamma) v+(1-1 / \gamma) v^{\prime}=z+(1-1 / \gamma) v^{\prime}$. Then $v^{\prime \prime} \in C$ and $v^{\prime \prime} \in z+H$ $=u+H$. Since each component of $C \backslash(z+H)$ is convex, the half open segment, $\left\{\beta v^{\prime}+(1-\beta) v^{\prime \prime} \mid 0<\beta \leqq 1\right\}$ lies entirely in the component of $C \backslash(z+H)$ which contains $C_{H}$ and hence no point of this set lies in $C \cap(u+C)$. Thus since $w \in C \cap(u+C)$ and since the points of the segment from $v$ to $v^{\prime}$ with $\alpha<1 / \gamma$ lie between $v^{\prime \prime}$ and $v^{\prime}, \alpha \geqq 1 / \gamma$. Hence $\alpha \gamma-1 \geqq 0$. Thus since $z, v^{\prime} \in C, w-z \in C$ and the entire ray from $\theta$ through $w-z$ is in $C$. Thus the ray from $z$ through $w$ lies in $C$. A similar argument shows that the ray from $z$ through $w$ also lies in $u+C$. Thus for any point $w \in C \cap(u+C)$ the ray from $z$ through $w$ lies in $C \cap(u+C)$. This shows that $C \cap(u+C)$ is a closed convex cone with vertex $z$. By Theorem $1, C \cap(u+C)=z+C$.

Theorem 3. Let $X, H, C_{H}$ be defined as in Theorem 2. Let $x, y \in X \backslash H$ be chosen in such a way that the line segment joining $x$ and $y$ intersects $C_{H}$ in a point distinct from $\theta$. Let $r_{x}, r_{y}$ be rays from $\theta$ through $x$ and $y$ respectively and let $C$ be the cone which is the convex hull of $r_{x}, r_{y}, C_{H}$. Let $L(x, y)$ be the two dimensional subspace of $X$ determined by $\theta, x, y$ and let $u \in L(x, y)$. Then there exists $z \in L(x, y)$ such that $C \cap(u+C)$ $=z+C$.

Proof. If $u \in C \cup(-C)$ then the theorem is obvious and $z=u$ or $z=\theta$. It can thus be assumed with no loss of generality that $u, x$ are 
in the same component of $X \backslash H$. Thus $r_{x} \cap\left(u+r_{y}\right)$ is nonvoid by construction. Let $z=r_{x} \cap\left(u+r_{y}\right)$. It will be shown as in the previous theorem that $C \cap(u+C)$ is a cone with vertex at $z$ and is hence equal to $z+C$. Let $w \in C \cap(u+C)$. It must be shown that the ray from $z$ through $w$ is in $C \cap(u+C)$. If $w \in z+H$ or $z+H$ separates $w$ from $H$, the same procedure as was used in Theorem 2 shows that $w-z \in C$. Since $u+C$ is a translate of $C, w-z+u \in u+C$ and the rays from $\theta$, $u$ through $w-z, w-z+u$ respectively lie in $C$ and $u+C$. Thus the ray from $z$ through $w$ also lies in $C$ and $u+C$ and hence in $C \cap(u+C)$. If $w$ lies on the other side of $z+H$, a similar argument can be applied to $u+C$ and $C$ and the ray from $z$ through $w$ lies in $C \cap(u+C)$. Thus $C \cap(u+C)$ is a cone with vertex $z$ and by Theorem $1, C \cap(u+C)$ $=z+C$.

CoROllaRy. Let a cone $C$ be the closure of the convex set determined by a set of rays $\left\{r_{\alpha}\right\}$ starting from $\theta$ and assume that these rays have the following properties. (a) the linear space spanned by $\left\{r_{\alpha}\right\}$ is dense in $X$, (b) if $r_{\alpha_{0}} \in\left\{r_{\alpha}\right\}$ there exists a second ray $r_{\alpha_{0}}^{\prime} \in\left\{r_{\alpha}\right\}$ such that the remainder of the rays in $\left\{r_{\alpha}\right\}$ generates a linear space whose closure is a hyperplane $H_{\alpha_{0}}$ which separates $r_{\alpha}$ and $r_{\alpha}^{\prime}$ and such that the two dimensional subspace determined by $r_{\alpha_{0}}$ and $r_{\alpha_{0}}^{\prime}$ intersects $C_{H_{\alpha}}=H_{\alpha} \cap C$. Then if $u$ is any point in the two space determined by any $r_{\alpha_{0}}$ and $r_{\alpha_{0}}^{\prime}$, there exists a $z \in X$ such that $C \cap(u+C)=z+C$.

ExAmple. Let $l$ be the space of all real summable sequences. Let $z_{i}^{+}$be the point $(0,0, \cdots, 0,1,0, \cdots), i=1,2,3, \cdots$ and $z_{i}$ $=(0,0, \cdots, 0,-1,0, \cdots), i=1,2, \cdots$ with 0 everywhere except in the $i$ th place. These points are then extreme points of the unit sphere in $l$. Let $C_{i}^{+}$be the cone with vertex $z_{i}^{+}$which is generated by the unit sphere. This is easily seen to be convex and closed in the norm topology. Then $C_{i}^{+}$satisfies the conditions of the preceding corollary since evidently if $r_{j}^{+}, r_{j}^{-}$are the rays from $z_{i}^{+}$through $z_{j}^{+}$, $z_{j}^{-}$respectively and $H_{j}$ is the closed subspace determined by the rest of the $r_{k}^{ \pm}, H_{j}$ is a hyperplane and $r_{j}^{+}, r_{j}^{-}$satisfy the conditions set forth there. Similarly for $C_{i}^{-}$for each $i$. Thus the unit sphere in $l$ is equal to $C_{i}^{+} \cap C_{i}^{-}$for any $i=1,2, \cdots$ where the $C_{i}^{+}, C_{i}^{-}$satisfy the conditions of the preceding corollary.

\section{REFERENCES}

1. J. A. Clarkson, $A$ characterization of $C$ spaces, Ann. of Math. vol. 48 (1947) pp. 845-850.

2. R. E. Fullerton, On a semigroup of subsets of a linear space, Proc. Amer. Math. Soc. vol. 1 (1950) pp. 440-442.

3. - A characterization of $L$ spaces, Fund. Math. vol. 38 (1951) pp. 127-136. 